\title{
Characterization of SnTe-doped InP grown by solid-source atomic layer molecular beam epitaxy
}

\author{
P. A. Postigo, ${ }^{\text {a) }}$ M. L. Dotor, F. García, D. Golmayo, and F. Briones \\ Instituto de Microelectrónica de Madrid (IMM-CNM-CSIC), Isaac Newton, \\ 8-PTM-Tres Cantos 28760 Madrid, Spain
}

(Received 3 August 2000; accepted for publication 7 November 2000)

\begin{abstract}
SnTe-doped InP layers were grown at low temperature by solid-source atomic layer molecular beam epitaxy. The samples were characterized by Hall measurements versus temperature, low temperature photoluminescence, $\mathrm{x}$-ray diffraction, and secondary ion mass spectroscopy. The temperature of the SnTe effusion cell was varied from 320 to $440{ }^{\circ} \mathrm{C}$, and the free electron concentration measured at room temperature ranged between $2.0 \times 10^{16} \mathrm{~cm}^{-3}$ and $5.6 \times 10^{18} \mathrm{~cm}^{-3}$ with the corresponding Hall mobility varying from 2320 to $1042 \mathrm{~cm}^{2} / \mathrm{V} \mathrm{s}$. (c) 2001 American Institute of Physics. [DOI: 10.1063/1.1337599]
\end{abstract}

\section{INTRODUCTION}

The increasing widespread use of InP-based devices in lightwave communication systems has resulted in a recent uptake in research on InP and related compound semiconductor materials. InP grown by solid-source molecular beam epitaxy (MBE) has been $n$-type doped with $\mathrm{Si}^{1,2}$ and $\mathrm{Sn}^{3}$ up to $\sim 1 \times 10^{20} \mathrm{~cm}^{-3}$. SnTe doping is compatible with the growth of InSb, ${ }^{4} \mathrm{GaInSb},{ }^{5}$ and $\mathrm{GaAs}^{6,7}$ and it has been applied to the growth of InP by organometallic vapor phase epitaxy ${ }^{8}$ but its effect has never been studied on InP grown by solid-source MBE. The SnTe source operates at low temperature (LT) $\left(<450{ }^{\circ} \mathrm{C}\right)$ and $\mathrm{Te}$ is expected as the main source of donors, whereas $\mathrm{Sn}$ is expected to act as a weakly compensating donor due to its amphoteric nature. In this article, we study the electrical and optical characteristics of SnTe-doped InP grown at LT $\left(<400{ }^{\circ} \mathrm{C}\right)$ by solid-source atomic layer MBE (ALMBE). Room temperature (RT) Hall measurements showed $n$-type doping in all of our SnTedoped InP with concentrations of carriers ranging from 2.0 $\times 10^{16}$ to $5.6 \times 10^{18} \mathrm{~cm}^{-3}$. Double crystal x-ray diffraction (XRD) was used to assess the structural quality of the samples. LT photoluminescence (PL) spectroscopy showed a blueshift of the band-to-band peak with increasing levels of doping. Secondary ion mass spectroscopy (SIMS) analysis showed very similar concentrations of $\mathrm{Sn}$ and $\mathrm{Te}$, both in the order of the total free carrier concentration at RT. Surprisingly, a very high concentration of oxygen was found also by SIMS. With the exception of the sample with the highest doping level $\left(n_{300 \mathrm{~K}}=5.6 \times 10^{18} \mathrm{~cm}^{-3}\right)$, the concentration of oxygen was found even higher than the concentrations of $\mathrm{Sn}$ and Te. Despite this anomalous high concentration of oxygen, the values obtained for the carrier concentration and for the mobility are comparable to those obtained by Si-doped InP.

\footnotetext{
a) Author to whom correspondence should be addressed; electronic mail: aitor@imm.cnm.csic.es
}

\section{EXPERIMENT}

The layers were grown on Fe-doped (semi-insulating) (100) InP substrates by ALMBE using all solid sources. In the ALMBE mode of growth the group V beam is modulated by opening the valve of the group $\mathrm{V}$ cell only a fraction $t$ of the time $T$ needed for the deposition of one monolayer of InP, which is the inverse of the growth rate $\left(T=1 / r_{g}\right)$. The $\mathrm{P}$ effusion cell has a two-temperature zone for production of white $\mathrm{P}_{4}$ and a cracker section for the cracking to $\mathrm{P}_{2}$, and it was designed ${ }^{9}$ to allow operation in a pulsed mode by incorporating a fast-acting valve. We used the reflectance difference (RD) technique for in situ, real-time control of the growth. ${ }^{10}$ The RD signal monitors the variation of the surface chemistry ${ }^{11}$ during growth and allows providing of the exact amount of phosphorous needed to complete one monolayer of InP. This method has been successfully used to obtain optimum electrical and optical properties of unintentionally doped ${ }^{12}$ and Be-doped ${ }^{13}$ InP grown at LT by ALMBE. Under this automated optical control we only need to set a growth rate, previously calibrated by reflection high-energy electron diffraction (RHEED) oscillations, typically of one monolayer per second (ML/s).

The RD signal is used as the on/off trigger signal for the phosphorous cell. The cell is open only at time $t$, which is a fraction of the time $T$ needed for the completion of one atomic ML and effuses during this time $t$ the optimum amount of phosphorus needed for the completion of the InP monolayer. The phosphorus pulse amplitude [beam equivalent pressure (BEP)] for each pulse $t$ was in the order of $1.4 \times 10^{-6} \mathrm{mb}$ when the cell was closed (corresponding to the background pressure) and increased up to $2.5 \times 10^{-6} \mathrm{mb}$ when the cell was open (the In cell was always open). The BEP amplitude can be adjusted independently so the total dose of phosphorous per second (i.e., per monolayer) is completely controlled. RHEED was used also to monitor the surface reconstruction during growth. During the growth by ALMBE we observed an alternating RHEED pattern between a $(2 \times 1)$ corresponding to a P-stabilized surface to a $(2 \times 4)$ shortly after the $\mathrm{P}_{2}$ beam is shut off. The thickness of 


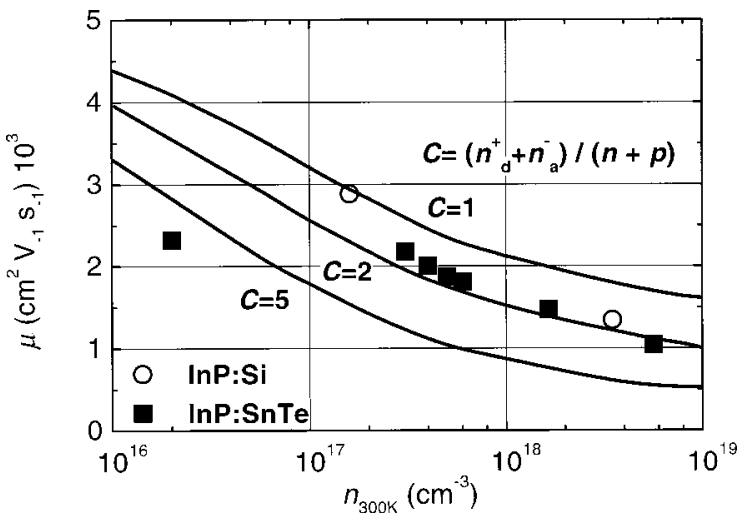

FIG. 1. Hall mobility at room temperature, $\mu_{300 \mathrm{~K}}$, as a function of the free-electron concentration measured at RT, $n_{300 \mathrm{~K}}$, for different SnTedoped InP layers. Open circles show two InP samples doped with $\mathrm{Si}$ for comparison.

the layers varied between 0.2 and $1 \mu \mathrm{m}$, depending on the doping level. The temperature of the SnTe effusion cell was varied in the range $320-440{ }^{\circ} \mathrm{C}$. Free carrier concentration $n$ and Hall mobility $\mu$ of the layers were measured in the temperature range of 20-300 K using the standard van der Pauw technique with contacts consisting of alloyed In balls. The densities of $\mathrm{Sn}$ atoms $\left(N_{\mathrm{Sn}}\right)$ and Te atoms $\left(N_{\mathrm{Te}}\right)$ as well as the density of oxygen atoms $\left(N_{\mathrm{O}}\right)$ incorporated during growth were measured by SIMS analysis with oxygen beam for four of the layers with different doping levels. The PL spectrum was measured by using an Ar laser, a close cycle helium cryostat, and a $22 \mathrm{~cm}$ monochromator with a Si photodiode.

\section{RESULTS}

Figure 1 shows Hall mobility at RT, $\mu_{300 \mathrm{~K}}$, as a function of the free-electron concentration measured at RT, $n_{300 \mathrm{~K}}$, for different SnTe-doped InP layers. For comparison, we have included also two InP samples doped with $\mathrm{Si}$. The values for the mobility of the SnTe-doped InP samples are comparable to those reported in the literature for Si-doped InP grown by solid-source MBE. ${ }^{1}$ Figure 1 shows also three $n_{300 \mathrm{~K}}-\mu_{300 \mathrm{~K}}$ curves for different compensation values for InP obtained from Ref. 14. By comparison with these curves we estimate that our SnTe-doped samples have a compensation factor, defined as $C=n^{+}{ }_{d}+n^{-}{ }_{a} / n+p$, slightly above $C=2$. Both $\mathrm{Si}$-doped InP samples are also close to this value. For the sample with minimum doping $\left(n_{300 \mathrm{~K}}=2.0\right.$ $\times 10^{16} \mathrm{~cm}^{-3}$ ) the compensation increases up to a value slightly below $C=5$.

Figure 2 shows the variation of the free-electron concentration with the temperature for several samples with different doping level. Even for the sample with the lowest doping level $\left(n_{300 \mathrm{~K}}=2.0 \times 10^{16} \mathrm{~cm}^{-3}\right)$ the behavior corresponds to that of conduction by the conduction band and the impurity band. ${ }^{15,16}$ For that sample, the temperature of the SnTe cell was $T_{\mathrm{SnTe}}=320^{\circ} \mathrm{C}$. We could not obtain samples with lower doping levels by decreasing the temperature of the SnTe cell below $320^{\circ} \mathrm{C}$. A fully degenerated behavior was found for

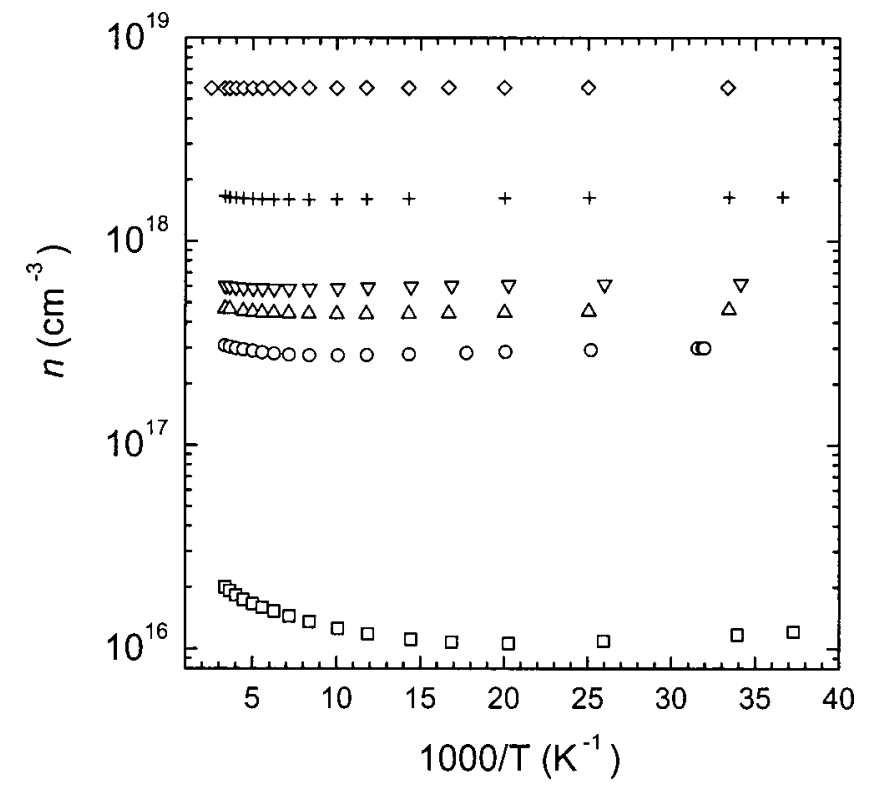

FIG. 2. Variation of the Hall electron concentration with the inverse of the temperature for several InP:SnTe samples with increasing doping level.

the sample with the highest doping level $\left(n_{300 \mathrm{~K}}=5.6 \times 10^{18}\right.$ $\mathrm{cm}^{-3}$ ) along the range of temperature measured $(20-300 \mathrm{~K})$. Figure 3 shows the variation of the Hall mobility with the temperature. As the doping level decreases the mobility shows an increasing tendency to drop for temperatures below 100-200 K, showing more clearly the effect of impurity scattering on carriers.

Figure 4 shows the concentration of $\mathrm{Sn}, \mathrm{Te}$, and $\mathrm{O}$ with depth obtained by SIMS for four of the samples. On all of them, the Te concentration runs almost flat along the profile whereas the Sn concentration profile shows a different path, with abrupt changes near the top and the bottom of the epilayer. The total amount of Sn remains approximately equal to the total amount of Te when added across all the profile. This behavior for the Sn agrees with an indication of Sn segrega-

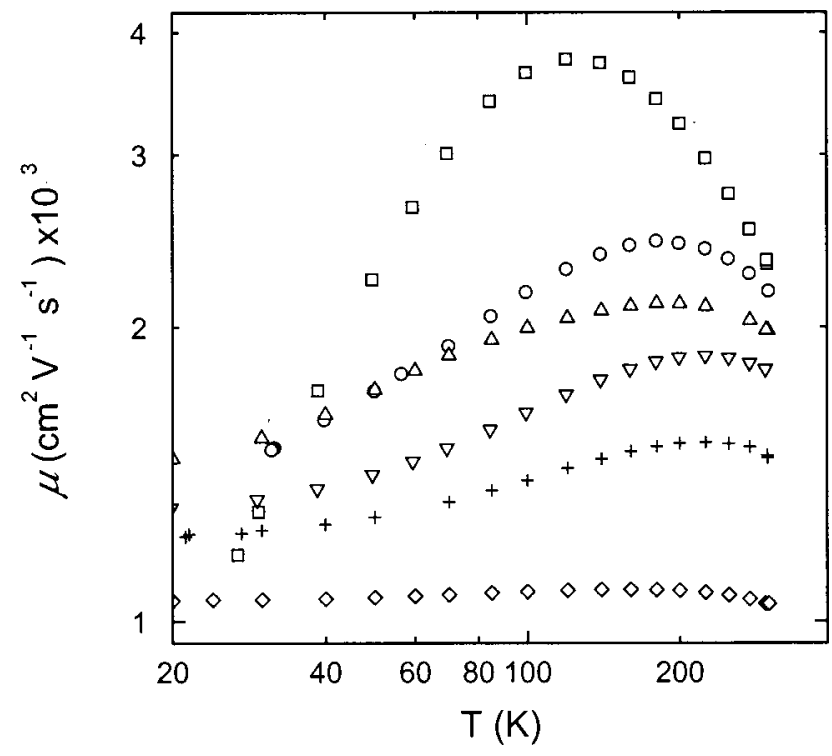

FIG. 3. Variation of the Hall mobility with the temperature of the samples showed in Fig. 2. 

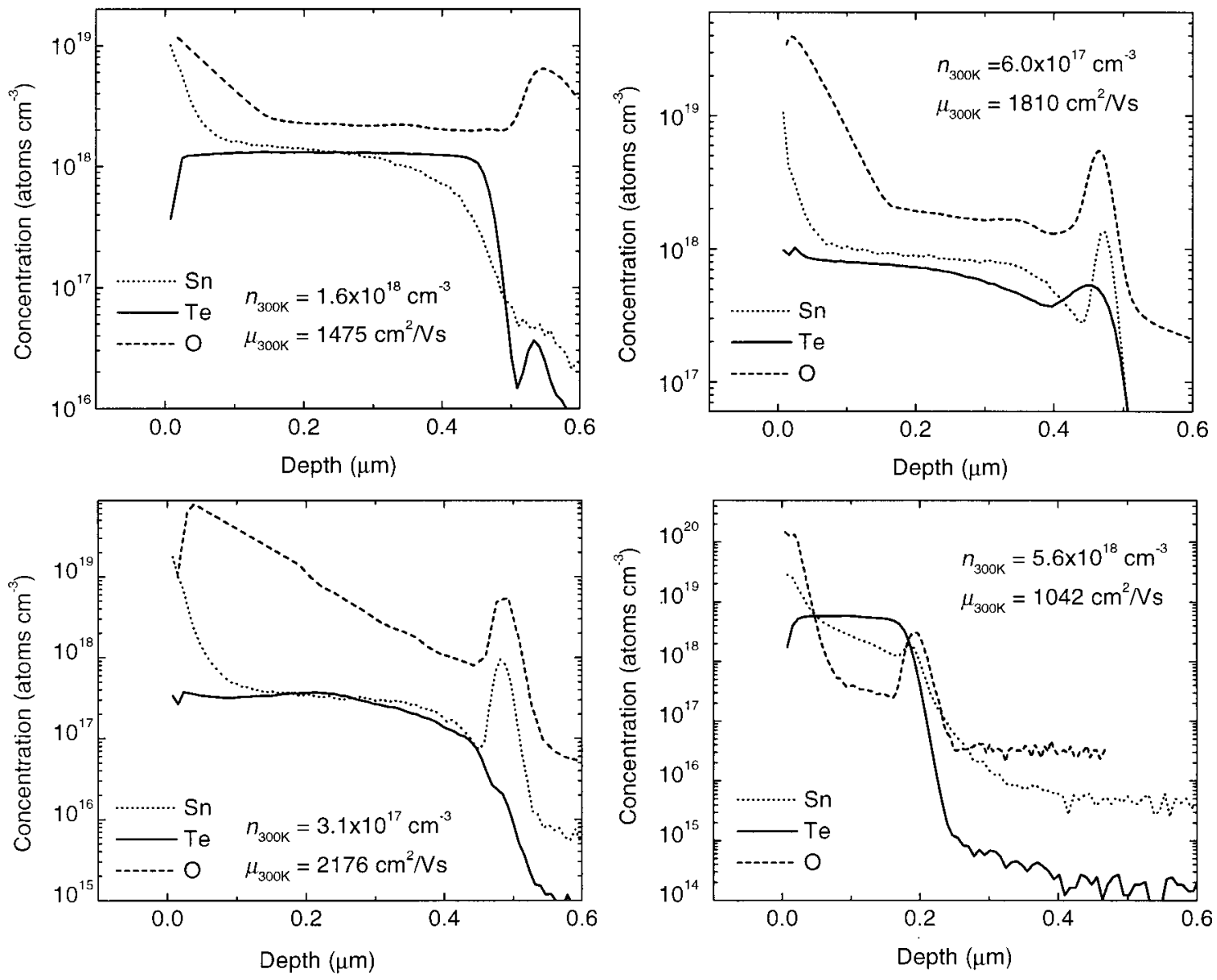

FIG. 4. Concentration of Sn, Te, and $\mathrm{O}$ as a function of depth obtained by SIMS for four of the InP:SnTe samples with increasing doping levels. Free electron concentration and mobility at RT for each sample are included. All the samples have a thickness of $\sim 0.4 \mu \mathrm{m}$ but the sample with highest doping $\left(n_{300 \mathrm{~K}}\right.$ $\left.=5.6 \times 10^{18} \mathrm{~cm}^{-3}, \mu_{300 \mathrm{~K}}=1042 \mathrm{~cm}^{-2} / \mathrm{V} \mathrm{s}\right)$ has $0.2 \mu \mathrm{m}$.

tion during the growth, despite the low temperatures used, as has been observed by other authors. ${ }^{3}$

The SIMS analysis shows also a high concentration of O in all of the four samples. Only the sample with the highest doping level $\left(n_{300 \mathrm{~K}}=5.6 \times 10^{18} \mathrm{~cm}^{-3}, \quad T_{\mathrm{SnTe}}=440^{\circ} \mathrm{C}\right)$ shows levels of $\mathrm{Sn}$ and Te higher than the level of $\mathrm{O}$ at depths far from the surface of the sample. High oxygen concentrations on phosphides grown by MBE have been reported by other authors ${ }^{17}$ and a dopant-tracking behavior has been observed in $\mathrm{InP}$ and $\mathrm{InGaP}$ layers doped with $\mathrm{Si}$ and Be ${ }^{18}$ In this case, however, the concentration of $\mathrm{O}$ diminishes as the $\mathrm{Sn}$ and Te concentration increases, opposite to the behavior observed for $\mathrm{Si}$ and Be. ${ }^{18}$ Despite the high levels of oxygen found in these four samples, the mobility does not show much lower values than the Si-doped ones, and the compensation factor is also similar for both sets.

Figure 5 shows the net free electron concentration at RT, $n_{300 \mathrm{~K}}$, the Sn and Te concentration obtained by SIMS, [Sn] and [Te], and the total concentration of $\mathrm{Sn}$ and $\mathrm{Te}$ atoms, $[\mathrm{Sn}]+[\mathrm{Te}]$, as a function of the reciprocal temperature of the SnTe furnace. The values for the concentrations were taken at the middle point of the total depth of the epilayer. The values for $n_{300 \mathrm{~K}}$ below $1.6 \times 10^{18} \mathrm{~cm}^{-3}$ exhibit a slope similar to the values from Collins et $_{\text {al. }}{ }^{7}$ for Sn Te doped GaAs.

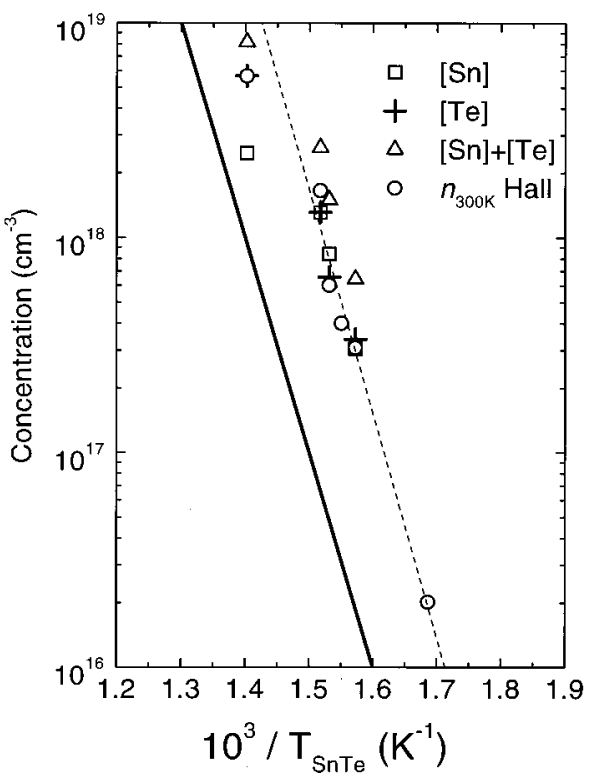

FIG. 5. Hall electron concentration at RT, $n_{300 \mathrm{~K}}$, Sn and Te concentrations obtained by SIMS, [Sn] and [Te], and total concentration of $\mathrm{Sn}$ and Te atoms $[\mathrm{Sn}]+[\mathrm{Te}]$ as a function of the reciprocal temperature of the SnTe cell. Dashed line indicates a linear fit of the data for $n_{300 \mathrm{~K}}$. Solid line represents the fit for $n_{300 \mathrm{~K}}$ data of SnTe-doped GaAs from Ref. 6. 

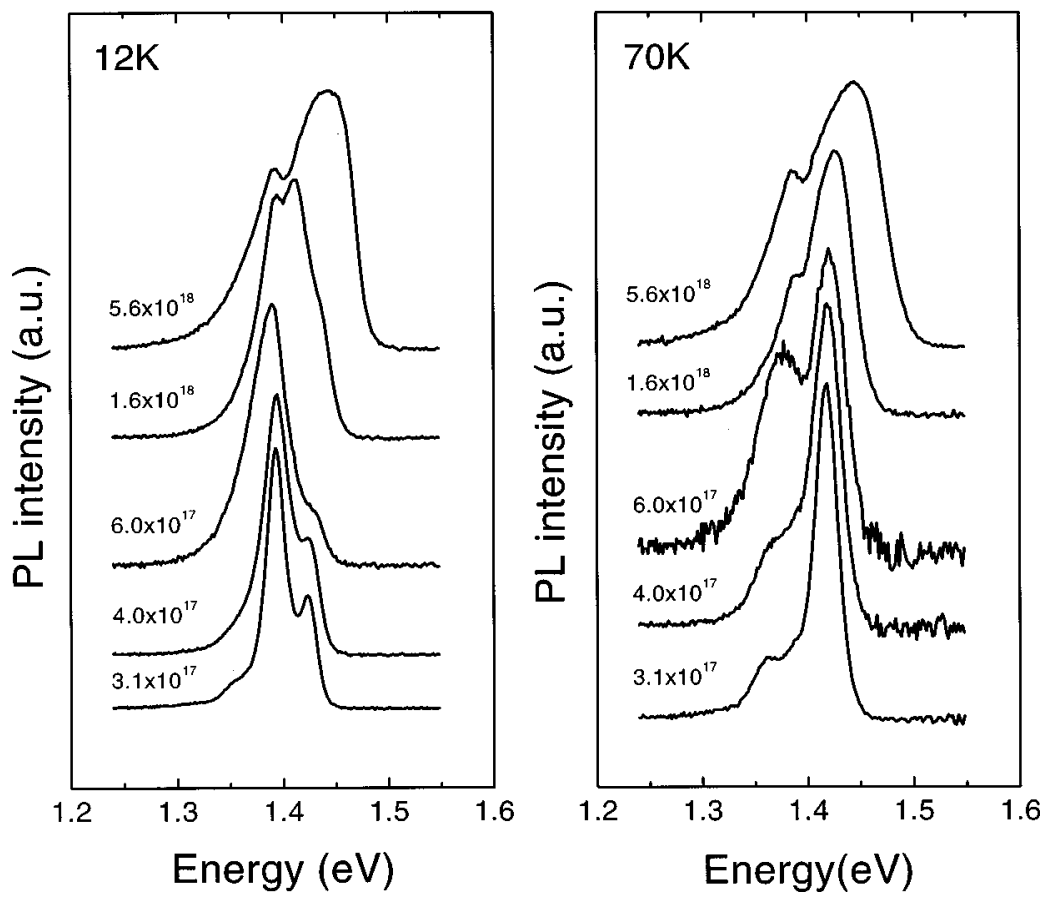

FIG. 6. PL spectra obtained at 12 and $70 \mathrm{~K}$ of the SnTe-doped InP samples with free electron concentration at RT ranging from $n_{300 \mathrm{~K}}=3.1 \times 10^{17} \mathrm{~cm}^{-3}$ to $n_{300 \mathrm{~K}}=5.6 \times 10^{18} \mathrm{~cm}^{-3}$.
The values for $n_{300 \mathrm{~K}}$ are under the $[\mathrm{Sn}]+[\mathrm{Te}]$ values, tracking the Te concentration closely. This may indicate that only the Te is responsible for the doping level reached, as has been observed in SnTe-doped $\mathrm{InSb}^{4}{ }^{4}$ while the $\mathrm{Sn}$ acts as an amphoteric dopant or it may be oxidized in form of $\mathrm{SnO}$. The sample with the highest doping level $\left(n_{300 \mathrm{~K}}=5.6\right.$ $\times 10^{18} \mathrm{~cm}^{-3}, T_{\mathrm{SnTe}}=440^{\circ} \mathrm{C}$ ) showed a clear deviation from the straight line suggesting that a degree of saturation was reached. A standard rocking-curve obtained by double crystal XRD on this sample showed a second peak near the substrate peak, indicating a clear lattice distortion due to the high doping level. During the growth of this sample a hazy RHEED pattern with slight three-dimensional features was observed and no samples were grown for temperatures of the SnTe above this value $\left(440^{\circ} \mathrm{C}\right)$.

Figure 6 shows the PL spectra obtained at 12 and $70 \mathrm{~K}$ of the SnTe-doped InP samples with free electron concentration at RT ranging from $n_{300 \mathrm{~K}}=3.1 \times 10^{17} \mathrm{~cm}^{-3}$ to $n_{300 \mathrm{~K}}$ $=5.6 \times 10^{18} \mathrm{~cm}^{-3}$. The intensity of the spectra was normalized to the maximum intensity of the highest peak. The good optical quality of the samples is evidenced by the absence of the band associated to stoichiometric defects at energies lower than the band gap. ${ }^{13}$ All of the spectra showed two main peaks: one near $1.42 \mathrm{eV}$ that corresponds to band-toband transitions $(\mathrm{B}-\mathrm{B})$ and other near $1.39 \mathrm{eV}$ that corresponds to a donor-band (D-B) or donor-acceptor (D-A) transitions. At $12 \mathrm{~K}$, for low carrier concentration (from $n_{300 \mathrm{~K}}=3.1 \times 10^{17} \mathrm{~cm}^{-3}$ to $n_{300 \mathrm{~K}}=6.0 \times 10^{17} \mathrm{~cm}^{-3}$ ) the main peak corresponds to impurity level transitions although the $\mathrm{B}-\mathrm{B}$ peak is clearly resolved, shifted $\sim 24 \mathrm{meV}$ to higher energies. Activation energies of $\sim 30$ and $\sim 6 \mathrm{meV}$ have been reported for Te-doped and Sn-doped GaAs samples respectively. ${ }^{19}$ As the doping level increases $\left(n_{300 \mathrm{~K}}=1.6\right.$ $\times 10^{18} \mathrm{~cm}^{-3}$ to $n_{300 \mathrm{~K}}=5.6 \times 10^{18} \mathrm{~cm}^{-3}$ ) the $\mathrm{B}-\mathrm{B}$ peak broadens, increasing its intensity and shifting to higher ener- gies, due to the increasing Fermi level and overlapping between the impurity band and the conduction band (BursteinMoss shift). ${ }^{20}$ The spectrum for the sample with $n_{300 \mathrm{~K}}=5.6$ $\times 10^{18} \mathrm{~cm}^{-3}$ showed a main peak corresponding to the $\mathrm{B}-\mathrm{B}$ transition. At $70 \mathrm{~K}$ a main $\mathrm{B}-\mathrm{B}$ peak dominates the spectra for all doping levels. The intensity of the peak associated to impurity levels, D-A and D-B, increases with the doping level. Both B-B and D-A/D-B peaks shift to higher energies and broad with the increasing doping level. Figure 7 shows the evolution of the energetic position of the B-B mean peak with the carrier concentration measured at RT. The peak shifts $\sim 25 \mathrm{meV}$ for each order of magnitude of increase in the carrier concentration. The typical BursteinMoss shift assuming an ideal parabolic conduction band should be $\sim 100 \mathrm{meV}$ for each order of magnitude in the carrier concentration. ${ }^{1}$ Increasing interband overlapping and

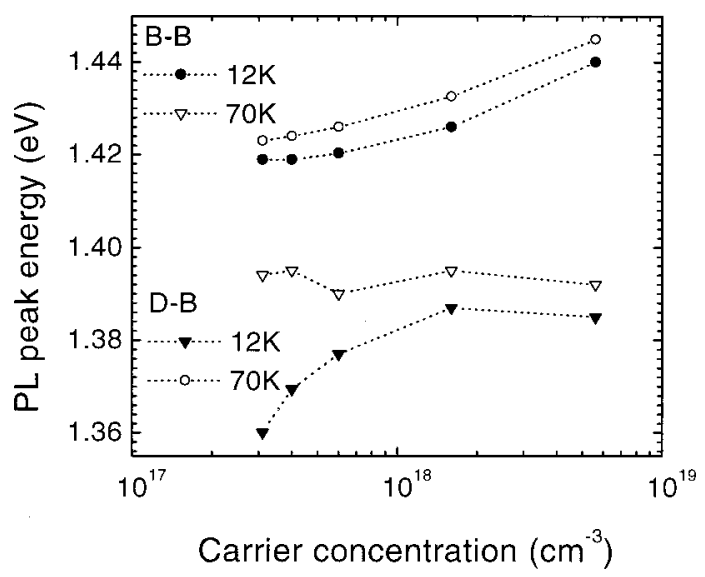

FIG. 7. Evolution of the energetic position of the B-B mean peak with the carrier concentration measured at RT. 
carrier degeneracy may be the reason for the slower increase that we observed. The D-B peak shows a different evolution as the carrier concentration increases. At $12 \mathrm{~K}$ the position of the $\mathrm{D}-\mathrm{B}$ (or $\mathrm{D}-\mathrm{A}$ ) peak does not vary with the carrier concentration, but at $70 \mathrm{~K}$ a clear blueshift is observed, reaching a saturation point for values of the carrier concentration higher than $1.6 \times 10^{18} \mathrm{~cm}^{-3}$. The higher thermal energy at this temperature is enough to allow the carriers transitions to slightly higher energies inside the overlapping bands.

In conclusion, we have successfully obtained $n$-type doping on InP samples using a SnTe source for dopant. Values of $n$ doping ranging from $2.0 \times 10^{16} \mathrm{~cm}^{-3}$ to $5.6 \times 10^{18}$ $\mathrm{cm}^{-3}$ with mobility ranging from 2320 to $1042 \mathrm{~cm}^{2} / \mathrm{V} \mathrm{s}$ at room temperature have been obtained. Despite the high concentration of oxygen observed in four of the samples, the structural, electrical, and optical properties obtained are comparable to the obtained for Si-doped InP samples. Further work is needed to understand the role of the Sn in the doping and the origin of the oxygen detected.

\section{ACKNOWLEDGMENTS}

The authors would like to thank Dr. A. J. Springthorpe, from Nortel Networks, for SIMS measurements, and the Spanish CICYT for financially supporting this research under Contract No. TIC-99-0645-C05-05.

${ }^{1}$ K. Radhakrishnan, H. Q. Zheng, P. H. Zhang, S. F. Yoon, and G. I. Ng, J. Cryst. Growth 204, 275 (1999).

${ }^{2}$ M. L. Dotor, D. Golmayo, A. Calle, J. R. Sendra, J. V. Anguita, L. Gonzalez, Y. Gonzalez, and F. Briones, Sol. Energy Mater. Sol. Cells 36, 271/ 276 (1995).
${ }^{3}$ M. B. Panish, R. A. Hamm, and L. C. Hopkins, Appl. Phys. Lett. 56, 2301 (1990).

${ }^{4}$ J. Li, T. A. Rawdanowicz, S. Iyer, S. Vankatraman, and W. J. Collins, Proceedings of the International Symposium 1998 TMS Annual Meeting, Warrendale, PA, 1998, pp. 277-281.

${ }^{5}$ J. F. Chen, S. H. Jaw, and A. Y. Cho, Jpn. J. Appl. Phys., Part 1 34, 81 (1995).

${ }^{6}$ M. Kuball, M. Cardona, A. Mazuelas, K. H. Ploog, J. J. Perez-Camacho, J. P. Silveira, and F. Briones, J. Appl. Phys. 77, 4339 (1995).

${ }^{7}$ D. M. Collins, J. N. Miller, Y. G. Chai, and R. Chow, J. Appl. Phys. 53, 3010 (1982).

${ }^{8}$ C. C. Hsu, J. S. Yuan, R. M. Cohen, and G. B. Stringfellow, J. Cryst. Growth 74, 535 (1986).

${ }^{9}$ Commercialized by RIBER Instruments SA Group.

${ }^{10}$ P. A. Postigo, M. L. Dotor, D. Golmayo, and F. Briones, Proceedings of the 8th European Workshop on Molecular Beam Epitaxy, Granda, Spain, 1995.

${ }^{11}$ P. A. Postigo, G. Armelles, T. Utzmeier, and F. Briones, Phys. Rev. B 57, 1362 (1998)

${ }^{12}$ P. A. Postigo, M. L. Dotor, F. García, P. Huertas, D. Golmayo, and F. Briones, J. Appl. Phys. 85, 6557 (1999).

${ }^{13}$ P. A. Postigo, M. L. Dotor, P. Huertas, D. Golmayo, and F. Briones, J. Appl. Phys. 77, 402 (1995).

${ }^{14}$ D. L. Rode, Phys. Rev. B 3, 1119 (1971).

${ }^{15}$ O. V. Emel'yanenko, T. S. Lagunova, D. N. Nosledov, and G. N. Talalaking, Sov. Phys. Solid State 7, 1063 (1964).

${ }^{16}$ H. Asahi, Y. Kawamura, M. Ikeda, and H. Okamoto, J. Appl. Phys. 52, $2852(1981)$.

${ }^{17}$ G. W. Wicks, M. W. Koch, F. G. Johnson, J. A. Varriano, and E. Kohnke, J. Vac. Sci. Technol. B 12, 1119 (1994).

${ }^{18}$ P. A. Postigo, G. Lullo, K. H. Choy, and C. G. Fonstad, J. Vac. Sci. Technol. B 17, 1281 (1999).

${ }^{19}$ A. G. Milnes, in Deep Impurities in Semiconductors (Wiley, New York, 1973); E. F. Schubert, Doping in III-V Semiconductors (Cambridge University Press, Cambridge, 1993).

${ }^{20}$ R. A. Smith, Semiconductors (Cambridge University Press, Cambridge, 1978). 